ACCEPTED MANUSCRIPT

\title{
Simulating partial discharge activity in a cylindrical void using a model of plasma dynamics
}

To cite this article before publication: George Callender et al 2018 J. Phys. D: Appl. Phys. in press https://doi.org/10.1088/1361-6463/aaedf0

\section{Manuscript version: Accepted Manuscript}

Accepted Manuscript is "the version of the article accepted for publication including all changes made as a result of the peer review process, and which may also include the addition to the article by IOP Publishing of a header, an article ID, a cover sheet and/or an 'Accepted

Manuscript' watermark, but excluding any other editing, typesetting or other changes made by IOP Publishing and/or its licensors"

This Accepted Manuscript is @ 2018 IOP Publishing Ltd.

During the embargo period (the 12 month period from the publication of the Version of Record of this article), the Accepted Manuscript is fully protected by copyright and cannot be reused or reposted elsewhere.

As the Version of Record of this article is going to be / has been published on a subscription basis, this Accepted Manuscript is available for reuse under a CC BY-NC-ND 3.0 licence after the 12 month embargo period.

After the embargo period, everyone is permitted to use copy and redistribute this article for non-commercial purposes only, provided that they adhere to all the terms of the licence https://creativecommons.org/licences/by-nc-nd/3.0

Although reasonable endeavours have been taken to obtain all necessary permissions from third parties to include their copyrighted content within this article, their full citation and copyright line may not be present in this Accepted Manuscript version. Before using any content from this article, please refer to the Version of Record on IOPscience once published for full citation and copyright details, as permissions will likely be required. All third party content is fully copyright protected, unless specifically stated otherwise in the figure caption in the Version of Record.

View the article online for updates and enhancements. 


\begin{abstract}
Partial discharge (PD) activity models typically use simplified descriptions of individual discharges to develop a model of discharge activity. This approach neglects the plasma dynamics of the discharge, and requires the use of multiple assumptions. In this work, plasma dynamic simulations of individual PDs are used to inform a PD activity model for discharges within a cylindrical cavity bounded by low density polyethylene (LDPE). Specifically, by considering the plasma dynamics of the discharges it is possible to determine: surface charge density distributions, apparent charges, the inception electric field and the residual electric field. The resulting PD activity model had only a single adjustable parameter, relating to the availability of seed charges, and was able to produce phase resolved PD (PRPD) patterns that were comparable with experimental data. Good agreement was also observed between the measured and simulated PD extinction voltage. The shortcomings of PD activity models are discussed including the poor understanding of the seed charge generation rate. Nevertheless, the model does allow robust conclusions on the PD dynamics in the experiment. The main contribution of this work is to show how simulations of plasma dynamics can be used to provide additional insight PD activity.
\end{abstract}

\title{
1 Introduction
}

Partial discharge (PD) is a term used to describe a localised breakdown event which does not bridge the gap between electrodes [1]. PDs frequently occur inside defects within insulation material in operational high voltage plant. PD measurements are therefore a commonly used tool to diagnose the health of electrical insulation and can provide insight into the remaining lifetime of electrical equipment [2]. At present simulating PD activity from operational high voltage plant is exceptionally difficult due to uncertainties in defect type, location and size. Instead models of PD activity have focused on replicating measurements taken from controlled experimental arrangements, in particular gaseous voids surrounded by solid dielectric material between parallel plate electrodes [3-6]. The work in this paper is intended to contribute to the research on PD activity modelling and in keeping with the literature a system consisting of a gaseous void surrounded by a solid dielectric material is considered.

PD activity modelling began with the foundational work of Niemeyer [3, 4]. In Niemeyer's work PDs were treated as instantaneous events that reduced the electric field inside a void to a residual value. The model used equations for free electron availability and factors to determine electric field distributions, which were based on the work of Crichton et al. [7, to simulate PD activity. Improvements in computational power meant that more recent PD activity models used numerical solvers to determine the electric field in a PD system [6, 8. Many of the fundamental assumptions remained the same as those introduced by Niemeyer. Earlier work simulating single PDs within spherical air-filled voids using a plasma dynamic model revealed that some of the concepts used in these models may be erroneous [9].

Models of plasma dynamics are a powerful tool for investigating breakdown in air, and are an established technique in the literature [10]. However, their use in investigating PD activity specifically is somewhat 
limited. In [11] a short sequence of PDs was simulated in a spherical void. Individual PDs in DC conditions have also been considered [12. To the author's knowledge the only example of a plasma dynamic model of a large number of PDs, such that it could be termed a PD activity model, is the work of Pan et al. 13 . In this work PDs occurring in an air-filled void that was bounded by a metallic electrode at one boundary and a dielectric material at the other boundaries. Although the work is a significant contribution it should be noted that: the results were not compared quantitatively against experimental data; photoionisation processes were treated as seed charges; the processes governing the generation of seed charge was not considered and the low order numerical schemes used, by necessity to reduce computational cost, could have impacted the simulation results.

The aim of this work is to develop a model of PD activity which is informed by plasma dynamic simulations of single discharges. It should be noted that this work has significant overlap with the literature on dielectric barrier discharges, which often use more complex models of plasma dynamics that capture chemistry and the dynamics electron energy distributions [14]. There are also similarities with work conducted by the plasma display panel community[15]. The emphasis in this work is to use a simple model which can give a reasonable description of the plasma dynamics of individual discharges to develop a model of PD activity. The intention is to provide insight into the qualitative plasma dynamics of PD in cylindrical voids during the initial stage of discharge activity, and to use the results from the model develop a PD activity model with a reduced number of free parameters.

\section{Experimental Arrangement}

The experimental data used in this work was generated during an investigation into ageing processes within cylindrical voids surrounded by low density polyethylene (LDPE) [16]. It is known that sustained PD activity leads to changes in the gaseous composition of the void and the surface chemistry of the void [17. Accurately capturing these changes using a model is considered beyond the scope of the work conducted here, and instead only the initial stages of PD activity are investigated using simulation.

20 samples were prepared for the experimental investigation in a chemical preparation room to reduce the chance of introducing imperfections and contamination. Each sample consisted of three films of LDPE $100 \mu \mathrm{m}$ thick with an area of $2500 \mathrm{~mm}^{2}$. A void with a diameter of $2 \mathrm{~mm}$ was created in the central film using a circular shaped micro drill bit. A pressing machine was then used to join the films, with a pressure of 0.145 bar at $90^{\circ} \mathrm{C}$ for 10 minutes. Once the sample was molded a micrometer was used to measure total thickness of the sample as $300 \mu \mathrm{m}$ to check that deformation of the sample had not taken place. A photograph of a typical sample is provided in Figure 1.

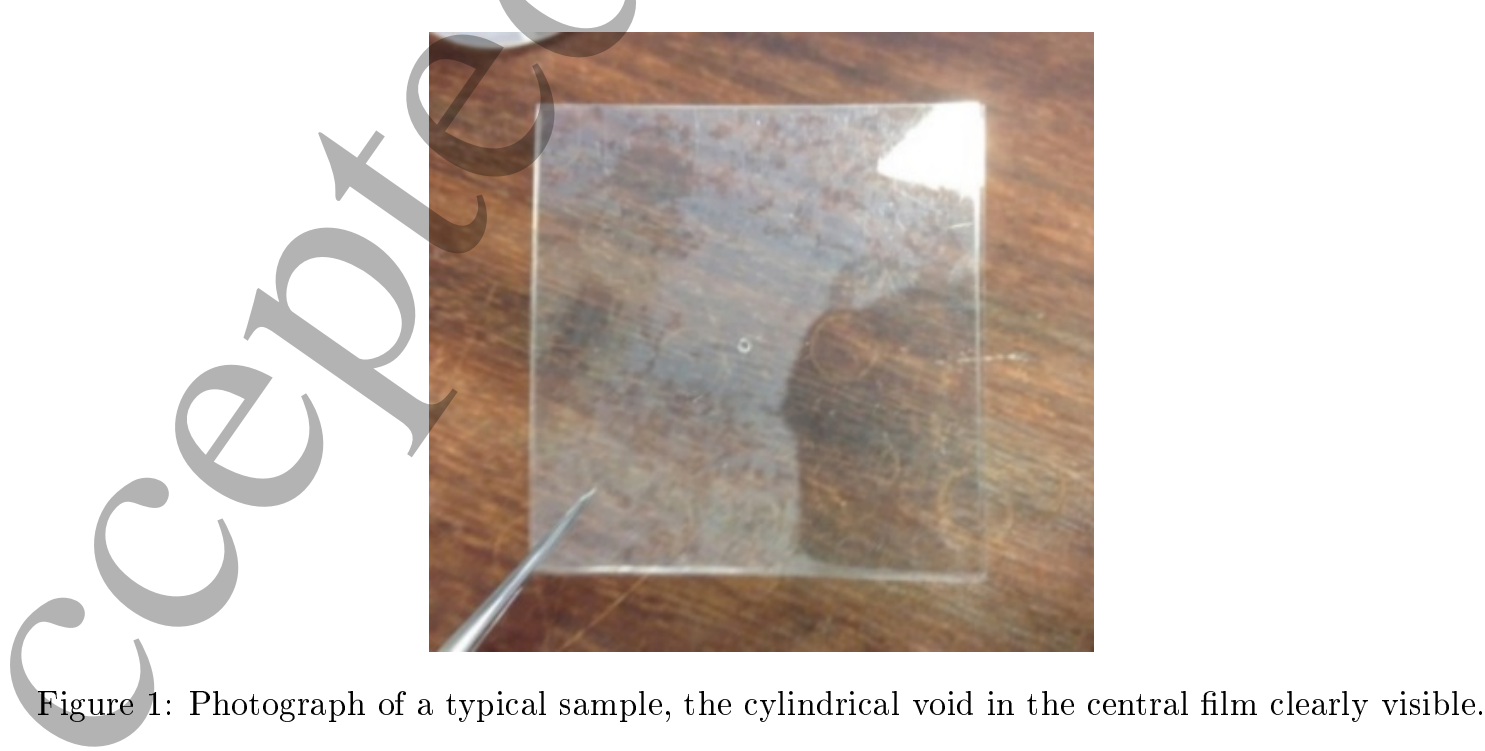

The samples were placed between brass electrodes in a silicone oil bath to prevent surface discharges. The top electrode was connected to a high voltage (HV) alternating current (AC) $50 \mathrm{~Hz}$ power supply. A 
digital signal oscilloscope in series with a coupling capacitor, both of which are parallel with the sample, is used to detect and measure PD activity within the void. PD measurement was calibrated in accordance with the IEC 60270 standard [18. This is a standard arrangement for measuring PD activity in samples of this nature [5, 19. Firstly the HV supply to the sample is gradually increased until PD activity is observed. The root mean square of the $\mathrm{AC}$ cycle required to initiate $\mathrm{PD}$ activity is referred to as the PD inception yoltage (PDIV) [18. PD measurements were then performed for 10 minutes, after which the applied voltage was decreased at a rate of $100 \mathrm{~V}$ per minute until PD activity was no longer observed. The root mean square of the AC cycle at which PD activity stops is referred to as the PD extinction voltage (PDEV) [18]. It is of note that the PDIV is significantly higher than the PDEV. In other words the very first PD requires a higher electric field in order to initiate, after which lower electric fields can sustain discharge activity. This could be due to the mechanism generating seed charge for the very first PD, which is generally assumed to be background radiation [4. After the first discharge the dominant form of seed charge generation is assumed to be surface emission from earlier discharges [4].

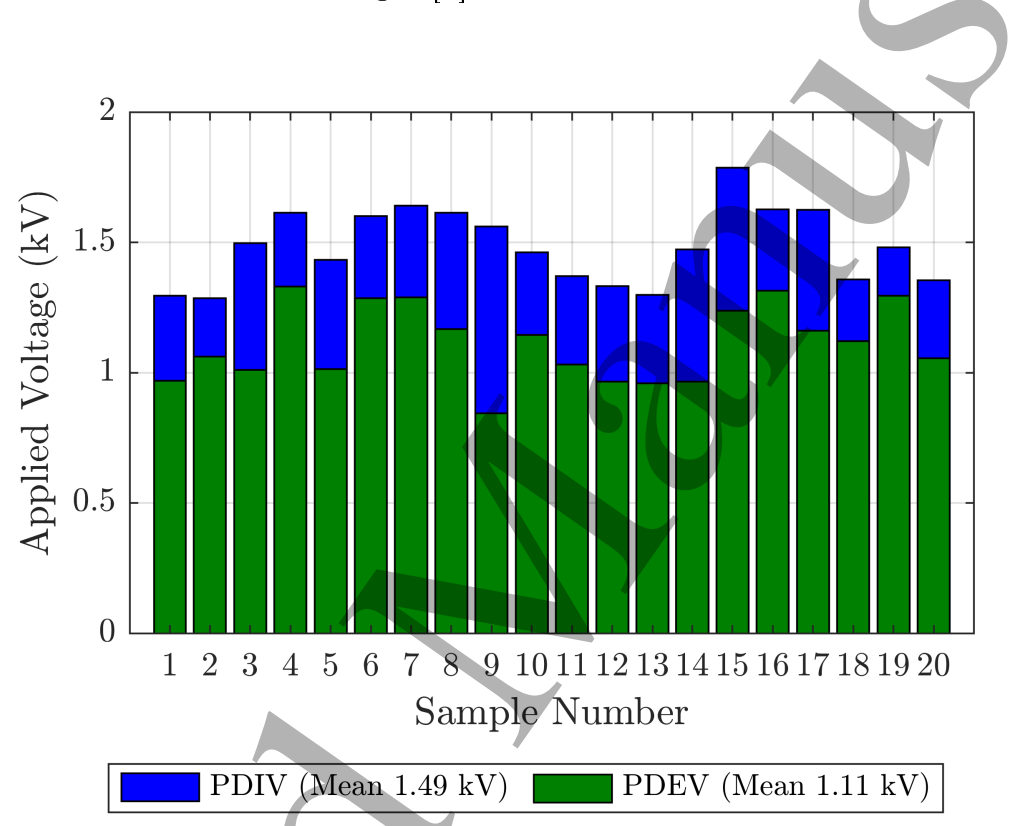

Figure 2: PDIV and PDEV (RMS values) for the 20 PD samples. All experiments were performed with a $50 \mathrm{~Hz}$ HV supply.

For each discharge two values are recorded:

1. The charge deployed at the measuring electrode, termed the apparent charge $q^{\prime}$, which for the samples under consideration is measured in $\mathrm{pC}$.

2. The time at which the discharge occurred is recorded, which is generally converted into an angle with respect to the $\mathrm{AC}$ cycle, termed phase angle $\theta$, measured in degrees.

These values are used to produce a phase resolved PD (PRPD) pattern, which is a scatter plot of apparent charge magnitude and phase angle. This is a standard technique for analysing PD activity [4, 19]. The PRPD patterns exhibit variation between samples, as shown in Figure 3. These differences are likely to be due to variations in the samples, despite efforts to keep them homogeneous, and varying environmental conditions. PD activity models have often used a large number of free parameters in order to produce a model that is in good agreement with a single experimental data set. However, for this work the data demonstrates that this is not possible, as there cannot be excellent agreement with all samples due to the inherent variability of the data sets.

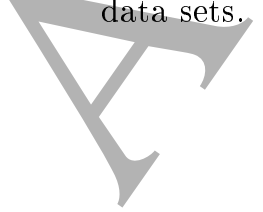




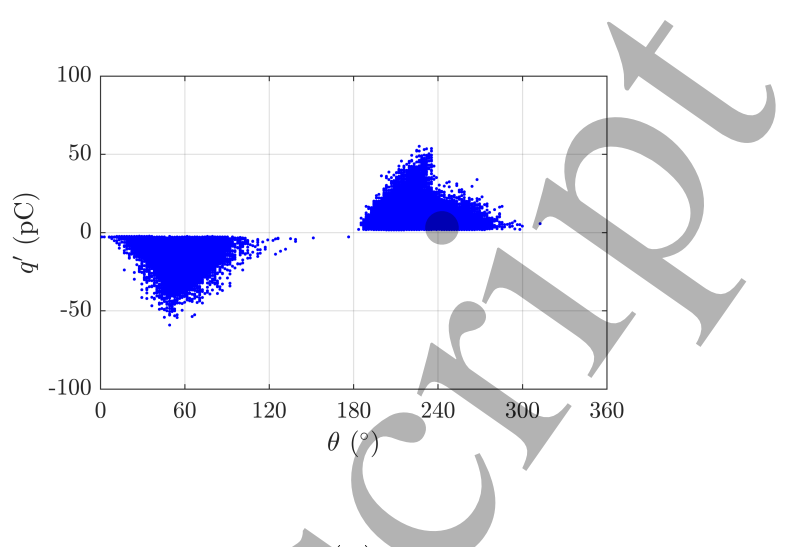

(a)

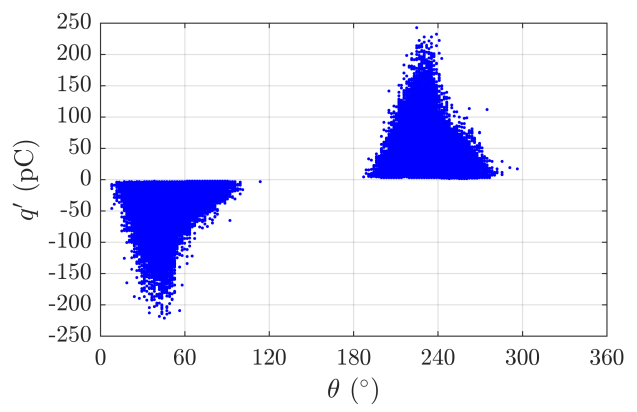

(c)

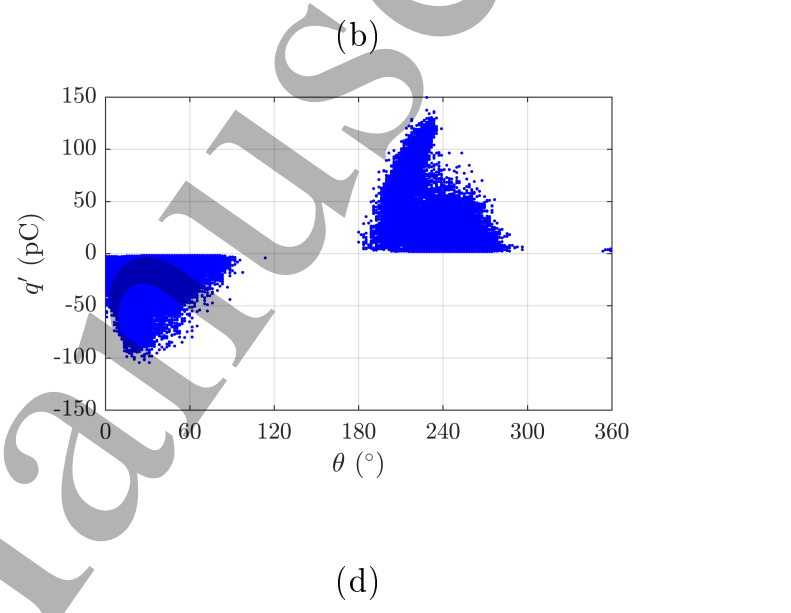

Figure 3: PRPD patterns of four different samples with a $50 \mathrm{~Hz}$ HV supply at PDIV: (a) Sample 2, (b) Sample 6 (c) Sample 8 and (d) Sample 11.

\section{Drift Diffusion Plasma Model}

The intention of the simulation work in this paper is to provide a qualitative explanation of discharge activity in terms of apparent charge magnitudes and PRPD patterns. As such a detailed description of plasma chemistry is considered to be beyond the scope of this work. Furthermore, only the initial stages of PD activity will be considered. To simulate PD after sustained discharge activity would require a more complex model as it would become necessary to develop a detailed description of the by-products formed on the void surface and the gaseous composition in the void. This would be difficult to achieve accurately and would likely necessitate making many unjustifiable assumptions. The approach taken here is to solve drift diffusion equations for electrons and "abstract" positive and negative ions to model plasma dynamics. This is an established technique to model plasma dynamics in air, and is still currently in use in the literature [20, 21. The model used here is identical to that used to simulate PD in spherical voids in earlier published work, [9], and as such only a brief overview is provided.

\subsection{Model Geometry}

The experimental arrangement consisted of three $100 \mu \mathrm{m}$ thick films of LDPE, with a hole drilled in the central film to create a void with a diameter of $2 \mathrm{~mm}$. In this work an axisymmetric geometry is used, shown in Figure 4. It is assumed that the contact between the LDPE films is sufficient to treat the LDPE as a single homogeneous region. A fillet with a $10 \mu \mathrm{m}$ radius is applied to the corners of the void in order to avoid the infinite electric field a sharp corner would cause. The simulation results show that at most voltages discharges spread along the majority of the top and bottom surfaces of the void, which is in agreement with both experimental and simulation work of discharges in cylindrical voids [12, 14]. Therefore, it is permissible 
to use an axisymmetric model, as off-axis discharges would still spread across the majority of the top and bottom void surfaces, and will give results that are qualitatively similar to those obtained in an axisymmetric system.

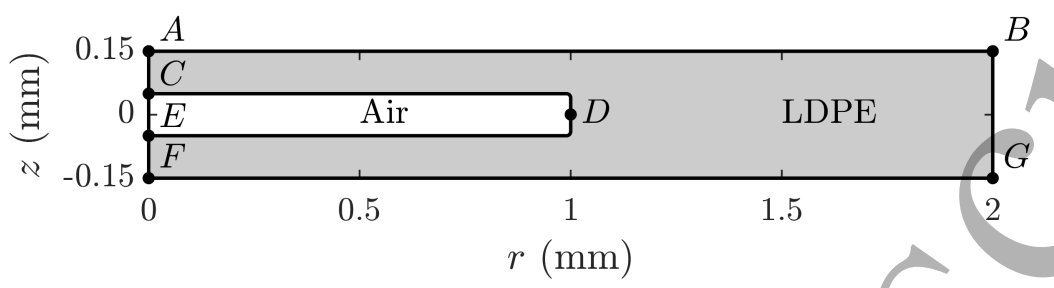

where $S_{\mathrm{ph}}$ is the photoionisation rate and the corresponding fluxes, $\vec{\Gamma}_{\mathrm{e}}, \vec{\Gamma}_{\mathrm{p}}$ and $\vec{\Gamma}_{\mathrm{n}}$, are

$$
\begin{aligned}
& \vec{\Gamma}_{\mathrm{e}}=n_{\mathrm{e}} \vec{W}_{\mathrm{e}}-D_{\mathrm{e}} \vec{\nabla} n_{\mathrm{e}} \\
& \vec{\Gamma}_{\mathrm{p}}=n_{\mathrm{p}} \vec{W}_{\mathrm{p}} \\
& \vec{\Gamma}_{\mathrm{n}}=n_{\mathrm{n}} \vec{W}_{\mathrm{n}}
\end{aligned}
$$

In equations (1) to $6 \sqrt{\mathrm{W}}, \vec{W}_{\mathrm{p}}$ and $\vec{W}_{\mathrm{n}}$ are the drift velocities for electrons, positive ions and negative ions respectively; $\alpha, \eta$ and $\beta$ are the ionisation, attachment and recombination coefficients respectively; $D_{\mathrm{e}}$ is diffusion coefficient for electrons. The swarm parameters for air are those introduced by Morrow and Lowke [22, the equations used to calculate them are provided explicitly in Appendix A. The three drift diffusion equations are coupled with the electrostatic equation to determine the electric field $\vec{E}$

$$
\nabla^{2} V=-\frac{e}{\varepsilon_{0} \varepsilon_{\mathrm{r}}}\left(n_{\mathrm{p}}-n_{\mathrm{e}}-n_{\mathrm{n}}\right)
$$

where $V$ is the electric potential, $e$ is the charge of an electron, $\varepsilon_{0}$ is the permittivity of free space, $\varepsilon_{\mathrm{r}}$ is the relative permittivity of the material $\left(\varepsilon_{\mathrm{r}}=1\right.$ in air and $\left.\varepsilon_{\mathrm{r}}=2.3 \mathrm{in} \mathrm{LDPE}\right)$ and

$$
\vec{E}=-\vec{\nabla} V
$$

The photoionisation rate $S_{\mathrm{ph}}$ is calculated using the three-exponential Helmholtz model developed by Bourdon et al. [23]. The drift diffusion equations, (1) to (6), and the Helmholtz equations to determine $S_{\mathrm{ph}}$ are only 
solved inside the void region. The electrostatic equation is solved within the entire model geometry, i.e. the void and the LDPE.

The boundary conditions for the drift diffusion equations at the void boundary are set based on the fluxes as follows

$$
\begin{aligned}
& \vec{n} \cdot \vec{\Gamma}_{\mathrm{e}}=\vec{n} \cdot\left(a_{\mathrm{e}} n_{\mathrm{e}} \vec{W}_{\mathrm{e}}-\gamma n_{\mathrm{p}} \vec{W}_{\mathrm{p}}\right) \\
& \vec{n} \cdot \vec{\Gamma}_{\mathrm{p}}=\vec{n} \cdot a_{\mathrm{p}} n_{\mathrm{p}} \vec{W}_{\mathrm{p}} \\
& \vec{n} \cdot \vec{\Gamma}_{\mathrm{n}}=\vec{n} \cdot a_{\mathrm{n}} n_{\mathrm{n}} \vec{W}_{\mathrm{n}}
\end{aligned}
$$

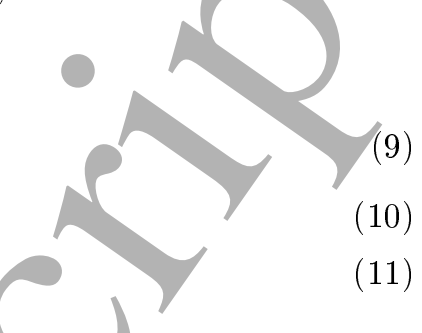

where $\gamma$ is the ion-impact secondary emission coefficient set to a token value of $0.001, \vec{n}$ is a unit normal vector facing outwards,

$$
a_{i}= \begin{cases}1 & \text { if } \operatorname{sgn}\left(q_{i}\right)=\operatorname{sgn}(\vec{n} \cdot \vec{E}) \\ 0 & \text { otherwise }\end{cases}
$$

$i=\mathrm{e}, \mathrm{p}$ and $\mathrm{n}, \mathrm{sgn}$ is the sign function and $q_{i}$ is the charge of the $i$ th species. The change in surface charge density due to the influx of charged species is also considered

$$
\sigma=\int_{0}^{t} e \vec{n} \cdot\left(n_{\mathrm{p}} \vec{W}_{\mathrm{p}}-n_{\mathrm{e}} \vec{W}_{\mathrm{e}}-n_{\mathrm{n}} \vec{W}_{\mathrm{n}}\right) d t
$$

The boundary conditions for the three Helmholtz terms are the same as those presented in earlier published work [9. The approach taken there was to set the boundary conditions such that the photoionisation rate calculated using the three exponential Helmholtz model was in good agreement with the photoionisation rate calculated using the full integral model of photoionisation in air proposed by Zhelezniak et al. [24]. The three exponential Helmholtz model solves three Helmholtz equations, with dependent variables denoted $S_{\mathrm{ph} i}$. The boundary conditions used for the Helmholtz equations were Diricet conditions on the first two components and a Neumann condition on the third component. In the earlier work it was verified that the agreement was reasonable for a spherical void with a radius of $0.5 \mathrm{~mm} \mathrm{[9].} \mathrm{In} \mathrm{this} \mathrm{work} \mathrm{a} \mathrm{different} \mathrm{void} \mathrm{geometry} \mathrm{is} \mathrm{considered,}$ so the verification process must be repeated. In order to compare the two models, referred to as 'Full Model' and 'Helmholtz' a Gaussian ionisation source is placed in the centre of the void. The photoionisation rate calculated using the two different methods is compared, see Figure 5. The parameters defining the Gaussian ionisation source and the boundary conditions used for the Helmholtz equations are identical to those used in earlier published work, $[9$. The results show that the photoionisation rate calculated using the Helmholtz model is the same order of magnitude as the photoionisation rate using the 'Full Model'. Order of magnitude changes to the photoionisation rate have not been found to significantly alter the model results, and it is sufficient for broadly capturing the plasma dynamics which is the purpose of this work. 


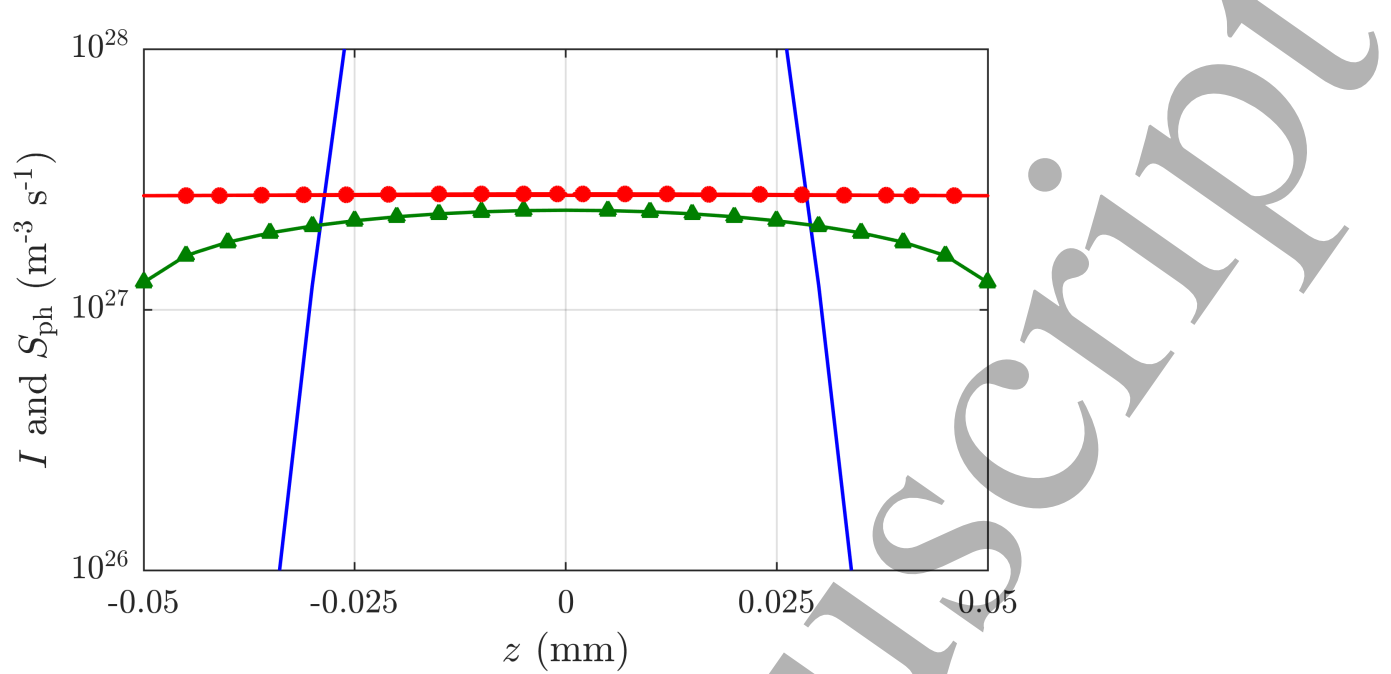

(a)

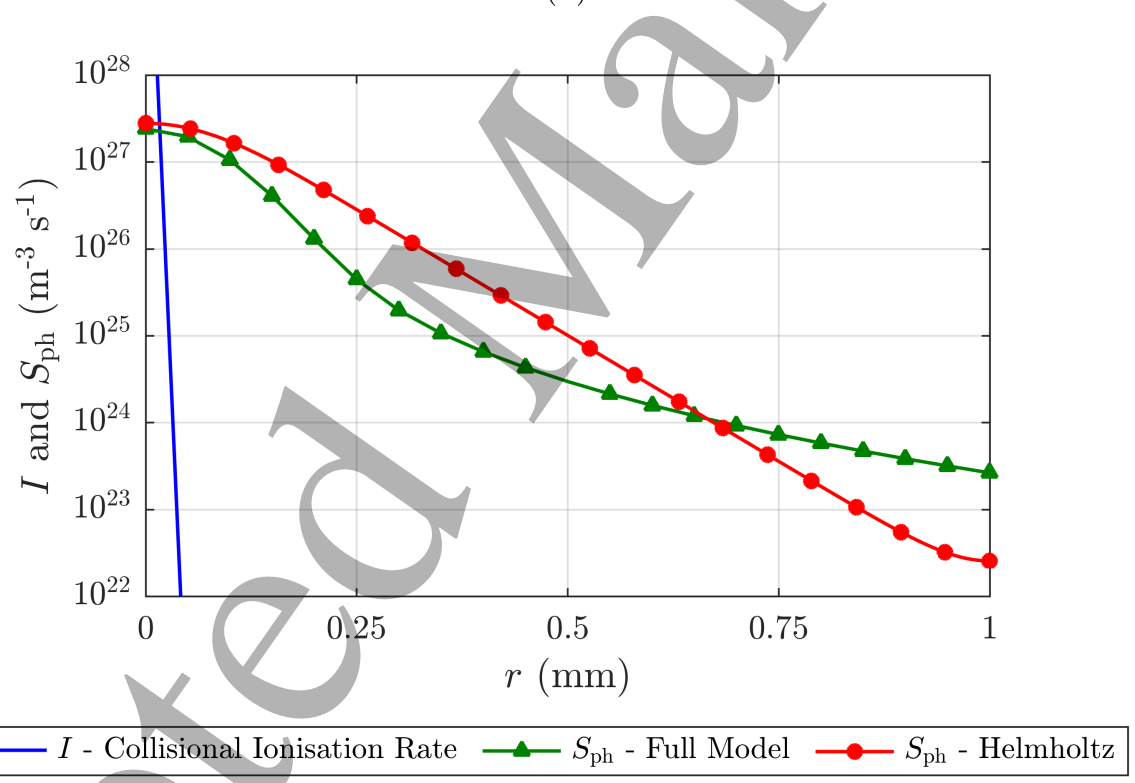

(b)

Figure 5: Comparison of photoionisation rate calculated using full model of photoionisation and Helmholtz model with modified boundary conditions. The Gaussian collisional ionisation production rate was centred at the origin: (a) photoionisation rates along the $z$ axis and (b) photoionisation rates along the $r$ axis.

The remaining boundary conditions are trivial, they are provided in Table 1 . 
Initial conditions are required for the drift diffusion equations. These take the form of a Gaussian seed of electrons and positive ions, with the initial number of negative ions set to zero. The plasma dynamic simulations are performed at a negative applied voltage, so the seed was centred at the top of the symmetry axis so that it had the maximum distance to multiply across the void and develop into a discharge. The initial conditions are explicitly

$$
\begin{aligned}
\left.n_{\mathrm{e}, \mathrm{p}}\right|_{t=0} & =n_{0} \exp \left(-\left(r^{2}+\left(z-z_{0}^{2}\right)\right) / s_{0}^{2}\right) \\
\left.n_{\mathrm{n}}\right|_{t=0} & =0
\end{aligned}
$$

where $z_{0}=50 \mu \mathrm{m}, n_{0}=10^{11} \mathrm{~m}^{-3}$ and $s_{0}=25 \mu \mathrm{m}$.

\subsection{Implementation}

The model is implemented using the built-in physics libraries in Comsol 5.3a [25], specifically: three transport of diluted species libraries to solve (1)-(3); the electrostatics library to solve (7); a coefficient form PDE library to solve the Helmholtz equations for $S_{\mathrm{ph}}$ and a boundary ODE library to solve (13). The implementation is identical to that used in earlier published work $\lfloor 9$. The numerical mesh was adapted based on the observed plasma dynamics by controlling the maximum element size. This led to mesh elements between $0.5 \mu \mathrm{m}$ and $2 \mu \mathrm{m}$ within the void region, with the smallest mesh elements close to the symmetry axis and the dielectric boundary. In the LDPE region the maximum element size was $25 \mu \mathrm{m}$. Halving the size of the mesh elements resulted in a change within $5 \%$ of the variables of interest.
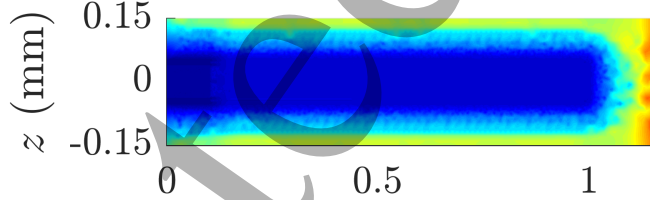

0.5

1

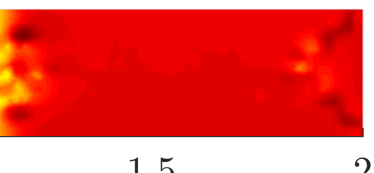

$r(\mathrm{~mm})$

1.5

\section{5}

10

15

\section{0}

Element Size $(\mu \mathrm{m})$

Figure 6: Surface plot of numerical mesh element size.

\section{Discharge Plasma Dynamics Simulations}

In this section the plasma dynamic simulations used to develop a PD activity model are discussed. The work consists of a series of DC discharge simulations in virgin voids, i.e. voids which have had no prior PD activity. The applied voltage is kept constant throughout the discharge because the discharges occur 
over the nanosecond to microsecond timescale, and the applied voltage AC cycle is $50 \mathrm{~Hz}$. The applied voltage, $V_{0}$, is set to negative values so that the measurable quantity of interest, apparent charge, is positive. All simulations consider the void to be filled with atmospheric pressure air. This is permissible during the initial stages of discharge activity. Sustained PD activity will result in significant changes to the gaseous composition, pressure and surface chemistry of the void, and would require a more sophisticated model.

The first investigation was performed to determine the inception electric field of the void. This is defined as the minimum electric field across the void required for a PD to take place [4. Note that this is somewhat confusing terminology, in that this electric field does not correspond to the discharge inception voltage PDIV. In fact it corresponds to the extinction voltage PDEV, the minimum voltage required for the PD to occur. This is because the PDEV is determined by gradually reducing the applied voltage until PD activity ceases. This means that there will be half cycles where PDs nearly completely neutralise all surface charge. This is supported experimentally by Pockel's cell measurements [26. Therefore the applied field alone, i.e. a virgin void scenario, must be sufficient at the peak of the waveform to initiate a PD. If it is not PD activity will cease, the applied voltage will be at PDEV. This is the reason why the simulated value is more representative of the PDEV than the PDIV.

The lowest voltage where the seed charge was found to develop into a PD, to the nearest $10 \mathrm{~V}$, was $-1.49 \mathrm{kV}$, which resulted in a PD with an apparent charge magnitude of $1.84 \mathrm{pC}$. At an applied voltage of $-1.48 \mathrm{kV}$ the apparent charge was several orders of magnitude lower and the plasma dynamics simply consisted of the seed charge being deployed into the void boundary. The inception electric field $E_{\text {inc }}$, corresponding to $V_{0}=-1.49 \mathrm{kV}$, is $7.97 \mathrm{kV} / \mathrm{mm}$. The applied voltage value of $-1.49 \mathrm{kV}$ calculated using simulation is in good agreement with the measured PDEV. In order to see this note that the PDEV values in Figure 2 are RMS values, the peak value across the $\mathrm{AC}$ cycle is higher by a factor $\sqrt{2}$. This means that for the samples measured the mean lowest applied voltage that could sustain PD activity was $\sqrt{2} \times 1.11=1.57 \mathrm{kV}$. The difference between these values is just over 5\% and the simulated PDEV is within the spread of PDEV values measured for the different samples, see Figure 2. It is acknowledged that the distribution of seed charge would influence the exact voltage at which a PD occurred. However, it should be noted that a parameter sweep of $n_{0}$ from $10^{9}$ to $10^{17} \mathrm{~m}^{-3}$ and $s_{0}$ from 1 to $50 \mu \mathrm{m}$ did not influence the voltage at which PD developed to within $10 \mathrm{~V}$. $\mathrm{PD}$ was found to initiate at $-1.49 \mathrm{kV}$ for all parameter combinations whereas it did not initiate at $-1.48 \mathrm{kV}$.

Another point of interest is that the commonly used expression for $E_{\text {inc }}$ for PD in voids is

$$
E_{\mathrm{inc}}=(E / p)_{\mathrm{cr}} p\left(1+\frac{B}{(p l)^{1 / 2}}\right)
$$

where $(E / p)_{\mathrm{cr}}=25.2 \mathrm{~V} \mathrm{~Pa}^{-1} \mathrm{~m}^{-1}, B=8.6 \mathrm{~Pa}^{1 / 2} \mathrm{~m}^{1 / 2}$ and $l$ is the length of the void in the direction of the electric field [27. (16) has been used to calculate inception electric fields for a range of PD systems including discharges in cylindrical and spherical voids [4]. The value of $E_{\text {inc }}$ calculated using (16) is $9.96 \mathrm{kV} / \mathrm{mm}$, which is in poor agreement with both measurement and simulation.

The plasma dynamics of the PD at inception consisted of diffusive avalanches. The discharge can be divided into the following stages:

(I) - The seed charge moved towards the bottom of the void undergoing multiplication, the transit across the void took approximately $0.5 \mathrm{~ns}$, see Figure $7 \mathrm{a}$. The space charge field was insufficient to significantly change the applied field throughout the discharge.

(II) - At the bottom of the void electrons were rapidly deployed into the bottom of the void surface as surface charge, see Figure 10. This resulted in a larger number of ions, both positive and negative, in the void compared to electrons. After multiplication positive ions moved back to the top of the void, and began to deploy positive surface charge, see Figure 8 b.

(III) - The deployed surface charge reduced the electric field in the vicinity of the symmetry axis. This led to increased ionisation off axis, due to the higher electric field, and resulted in the charged species spreading across the void surface, see Figure 9. Ionisation processes were not sufficient to sustain space charge in the void as the charged species moved into higher field regions, with charge deployed at the void surfaces off axis, see Figure 10.

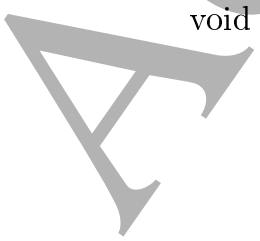




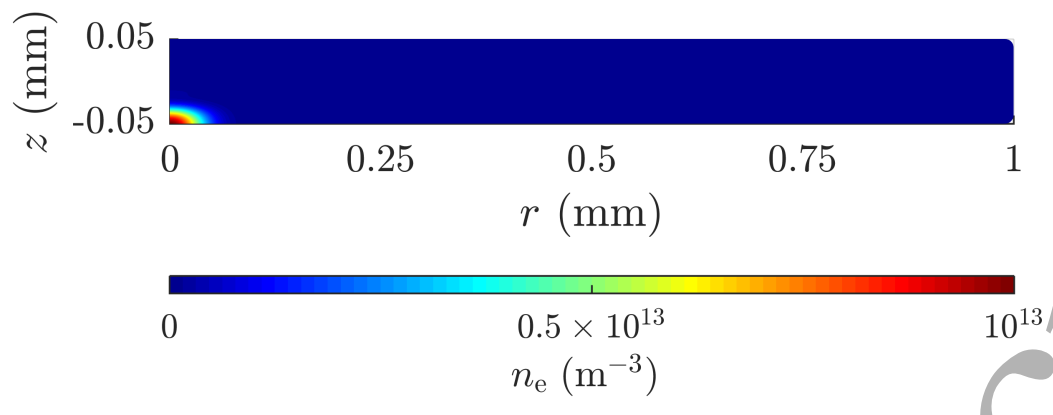

(a)
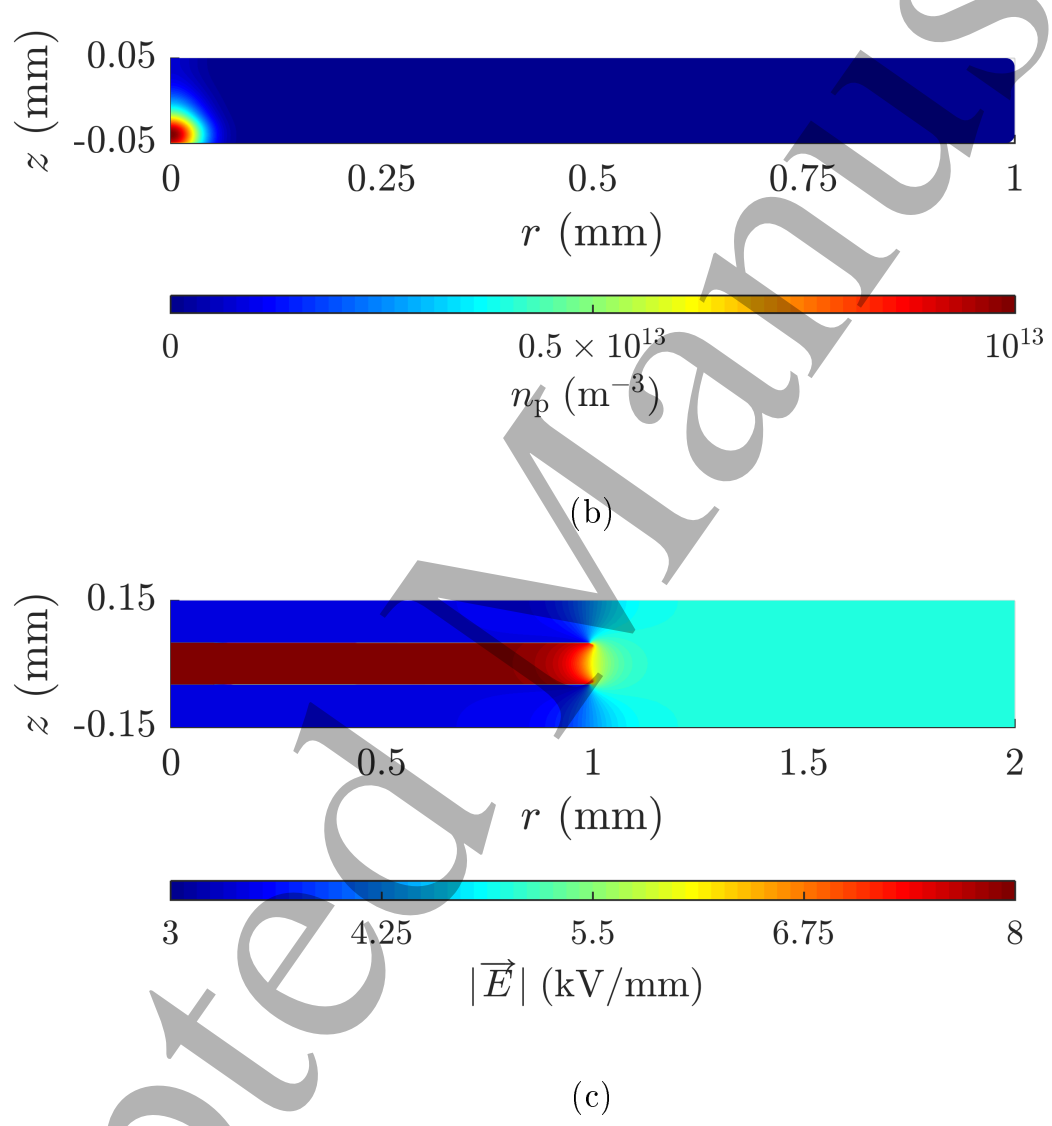

Figure 7: Discharge dependent variables for inception conditions at $0.4 \mathrm{~ns}$ (I) : (a) electron number density, (b) positive ion number density, (c) electric field magnitude. 


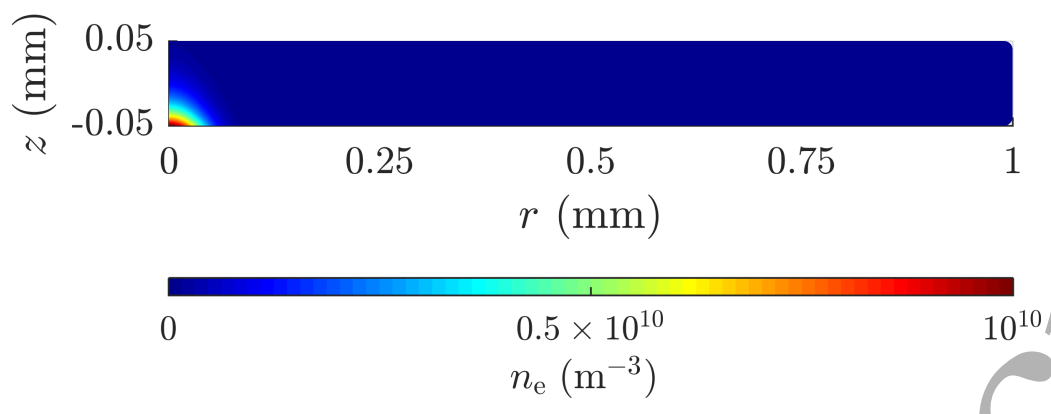

(a)
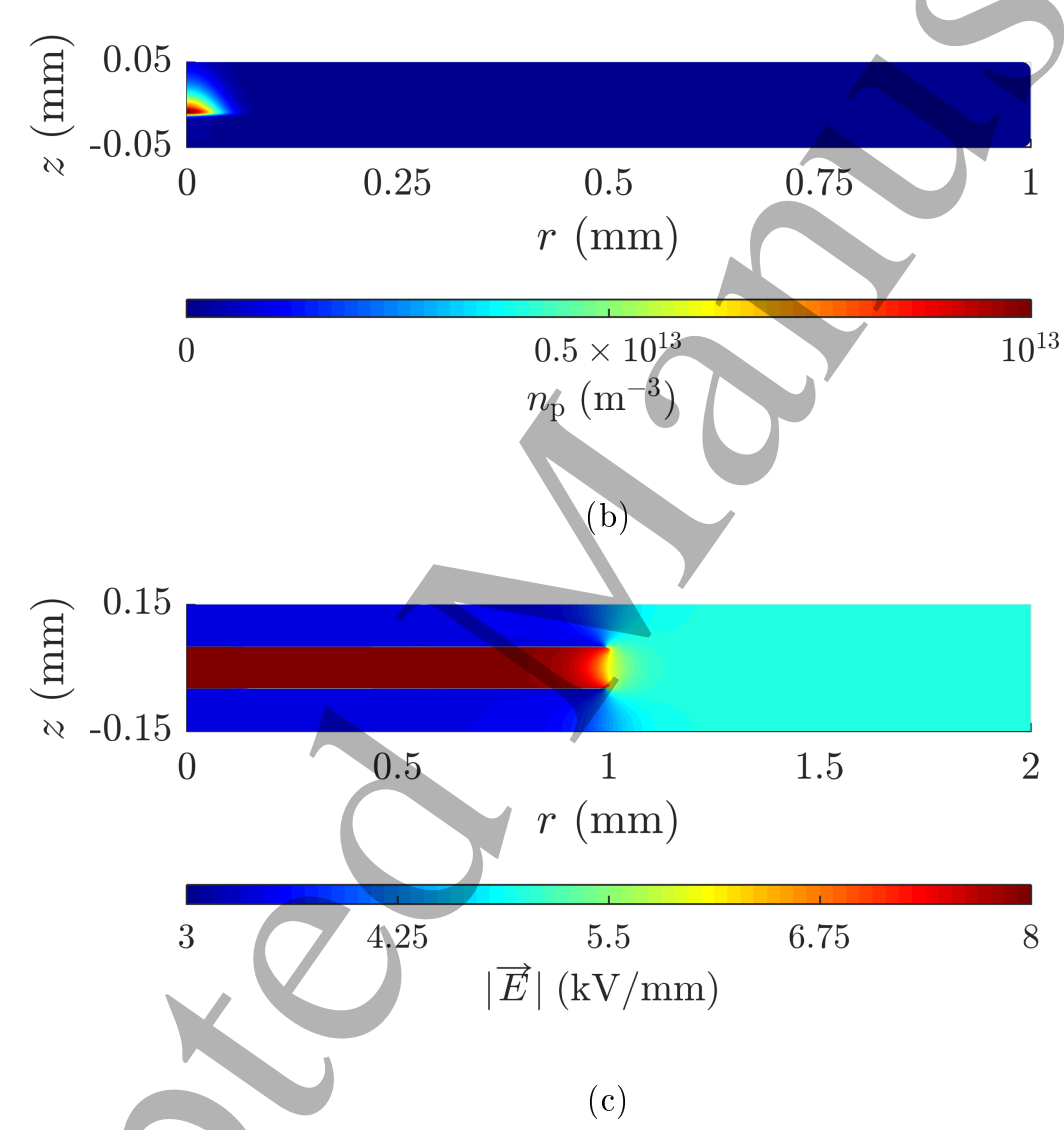

Figure 8: Discharge dependent variables for inception conditions at $20 \mathrm{~ns}$ (II): (a) electron number density, (b) positive ion number density, (c) electric field magnitude. 


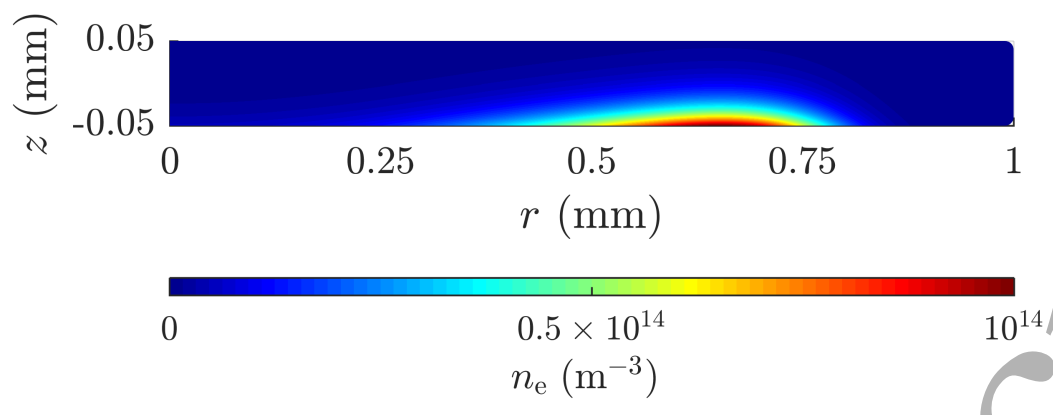

(a)
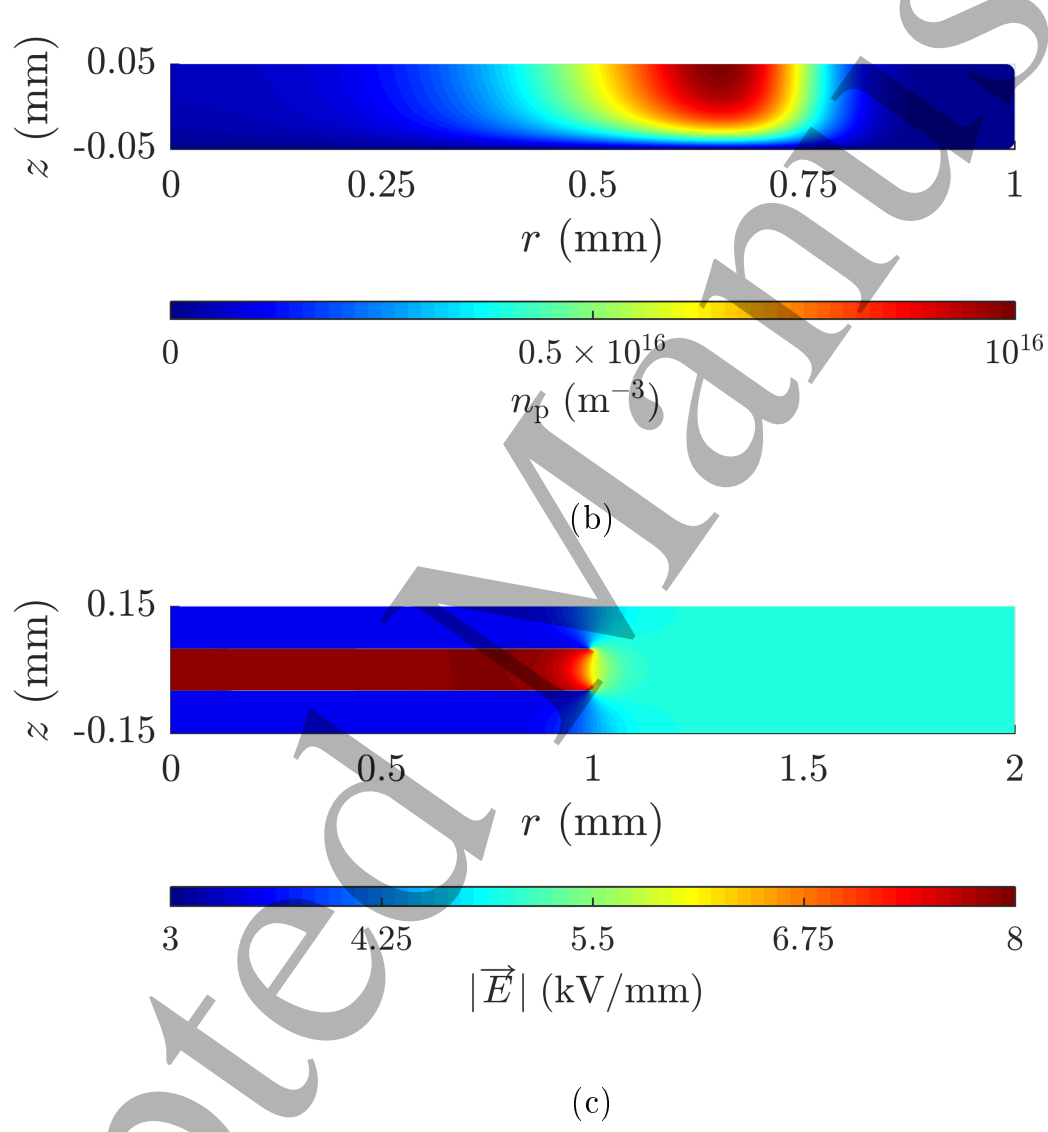

Figure 9: Discharge dependent variables for inception conditions at $10 \mu \mathrm{s}$ (III) (a) electron number density, (b) positive ion number density, (c) electric field magnitude. 


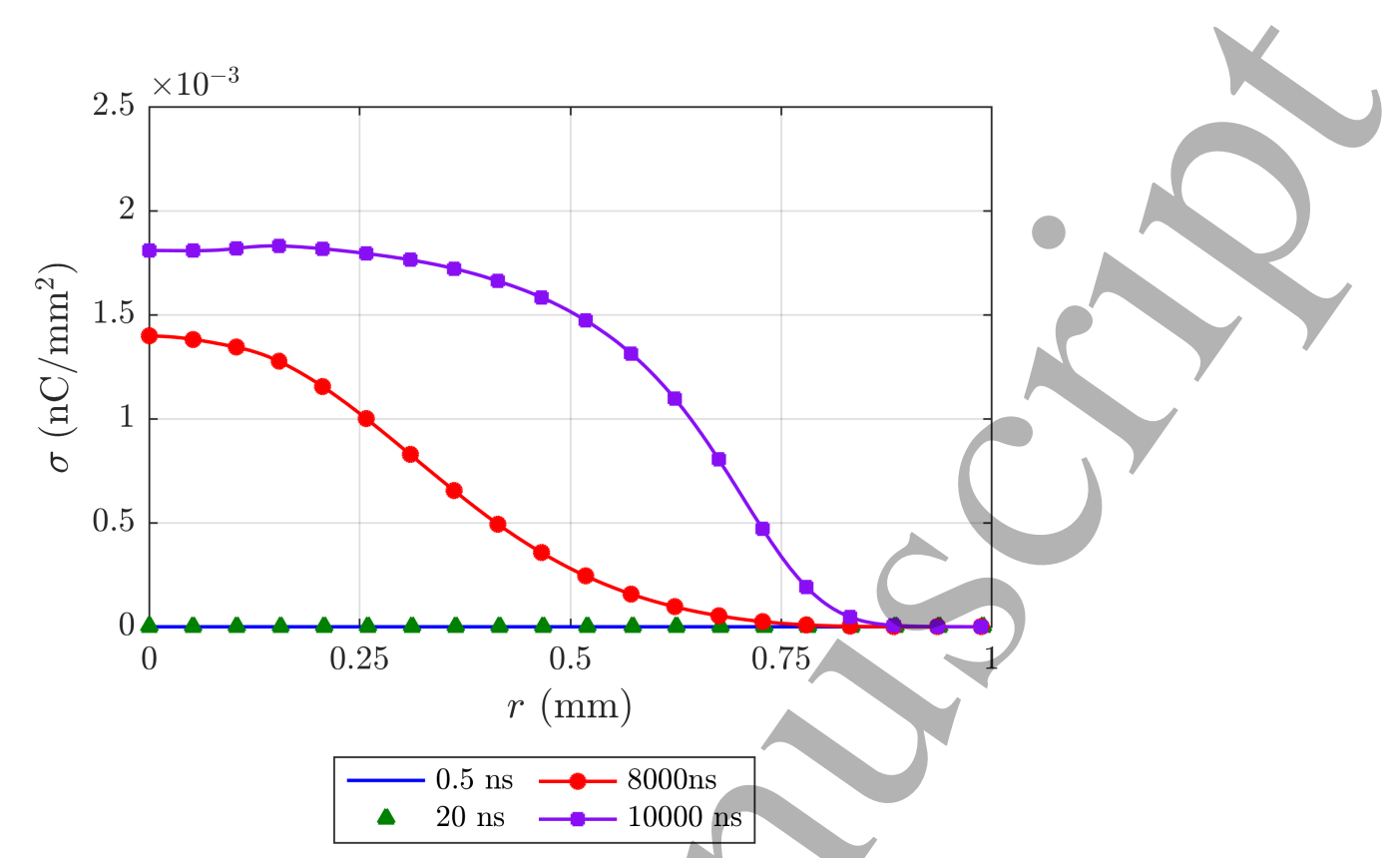

(a)

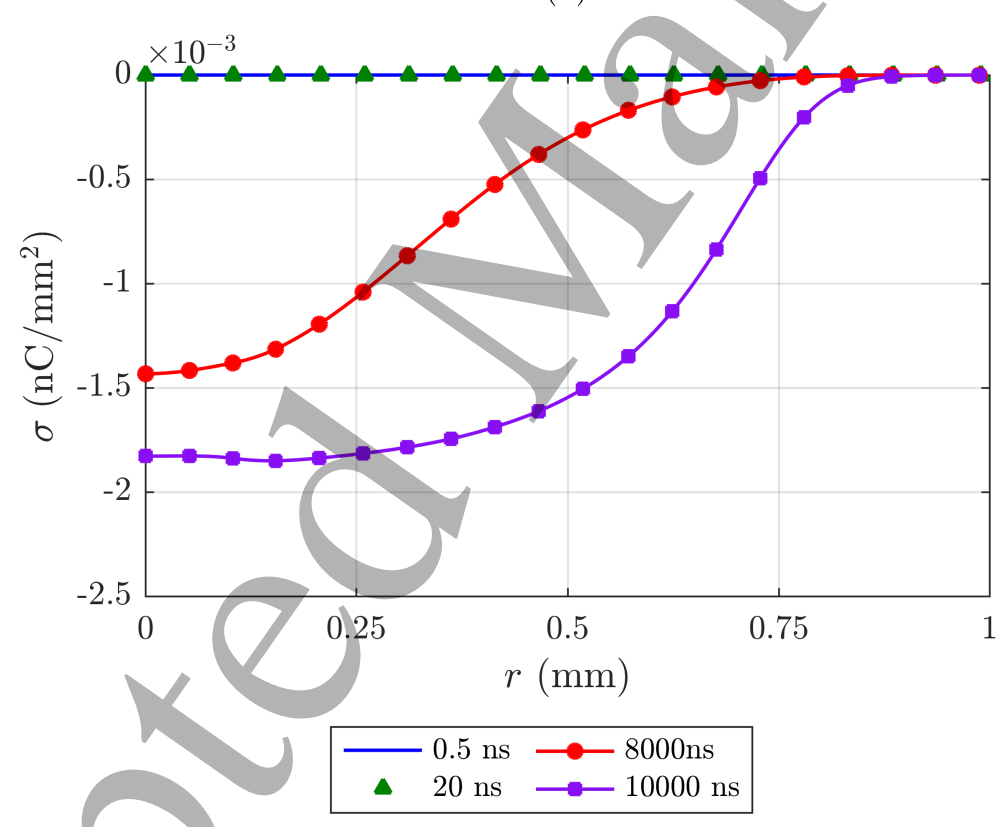

(b)

Figure 10: Surface charge density on the void surface for inception conditions at different times during the discharge: (a) top surface and (b) bottom surface.

In order to develop a PD activity model, discharges were simulated at increasing applied voltages until the apparent charge of the simulated PD exceeded the maximum measured PD. Simulations were performed at $100 \mathrm{~V}$ intervals between $-1.5 \mathrm{kV}$ and $-2.2 \mathrm{kV}$. Combined with the simulation at $1.49 \mathrm{kV} 9$ simulations were required to develop the PD activity model. Plasma dynamics were similar for all applied voltages, the main difference was that discharges occurred over a shorter timescale at higher voltages and deposited more surface charge, see Figure 11. Surface charge is deployed both by multiplication of the seed charge near the symmetry axis, (I) and by the spread of charge across the void surface, (II). This led to 'spots' of surface charge near the symmetry axis for some applied voltages, depending on the relative duration of the different 
stages. The surface charge density distribution near the symmetry axis was found to be a function of the seed charge distribution. However, it is important to note that changes to the seed charge distribution were not found to significantly impact the apparent charge or the electric field in the void after PD, known as the residual field. This is the key information taken from the plasma dynamic simulations to construct the PD activity model, which is the main contribution of this work.

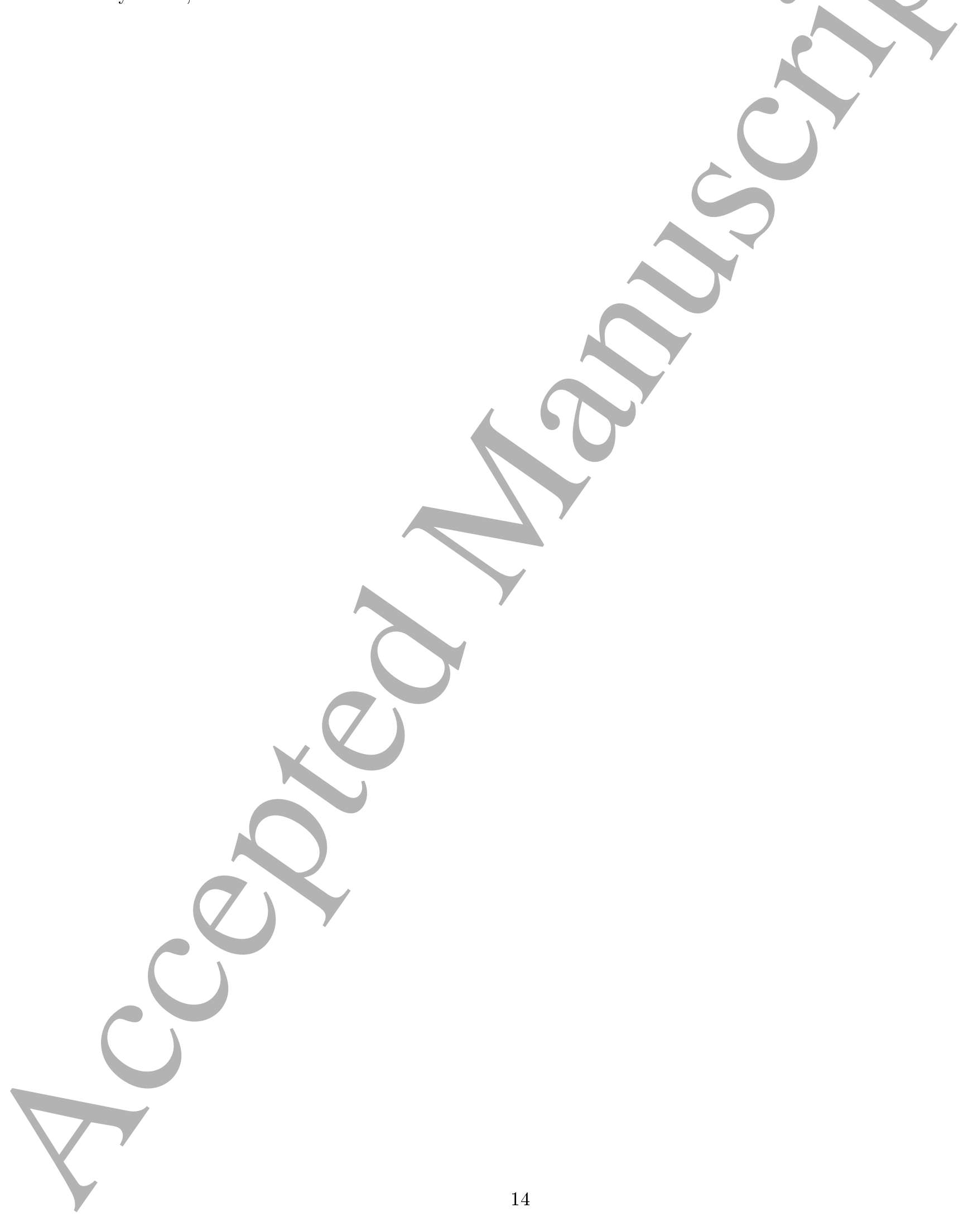




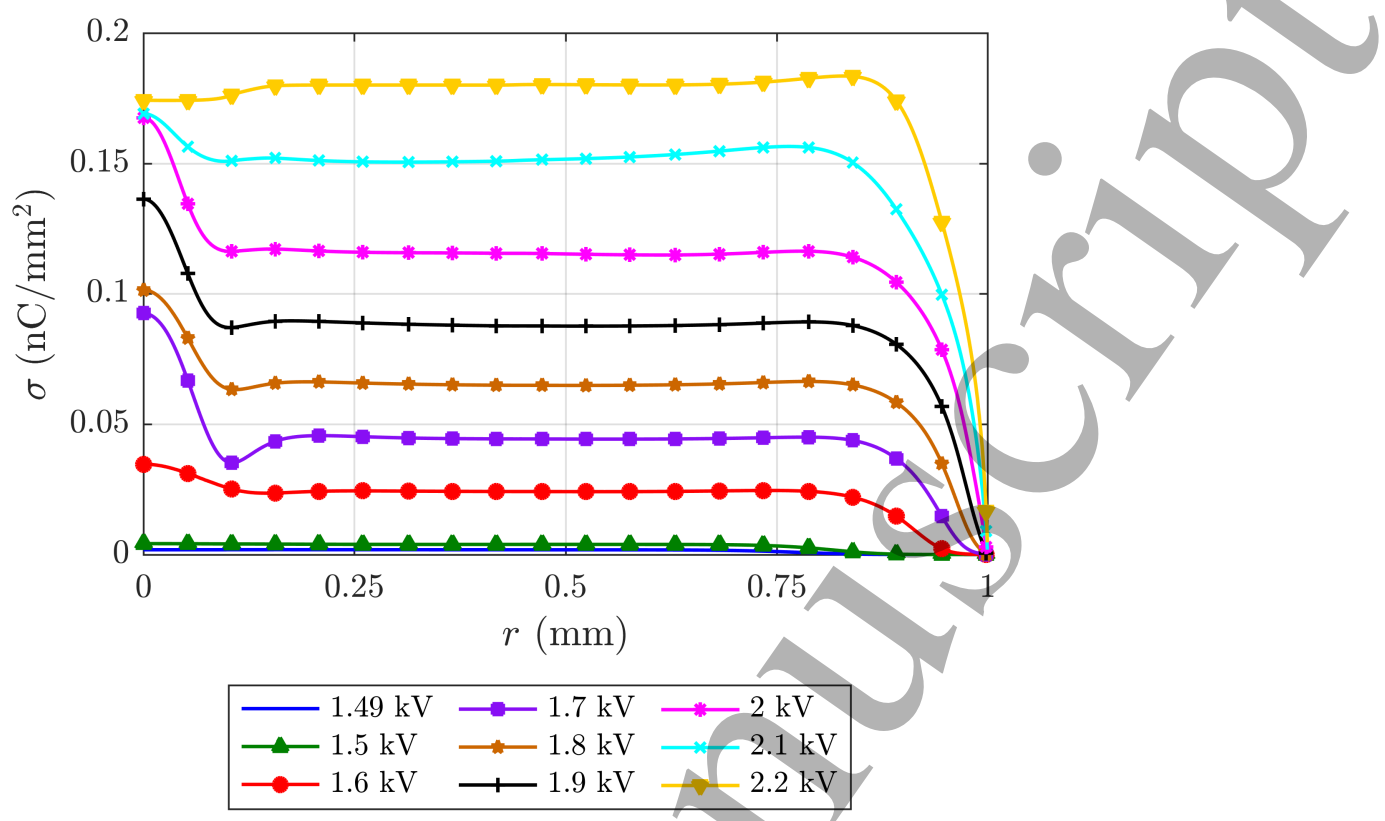

(a)
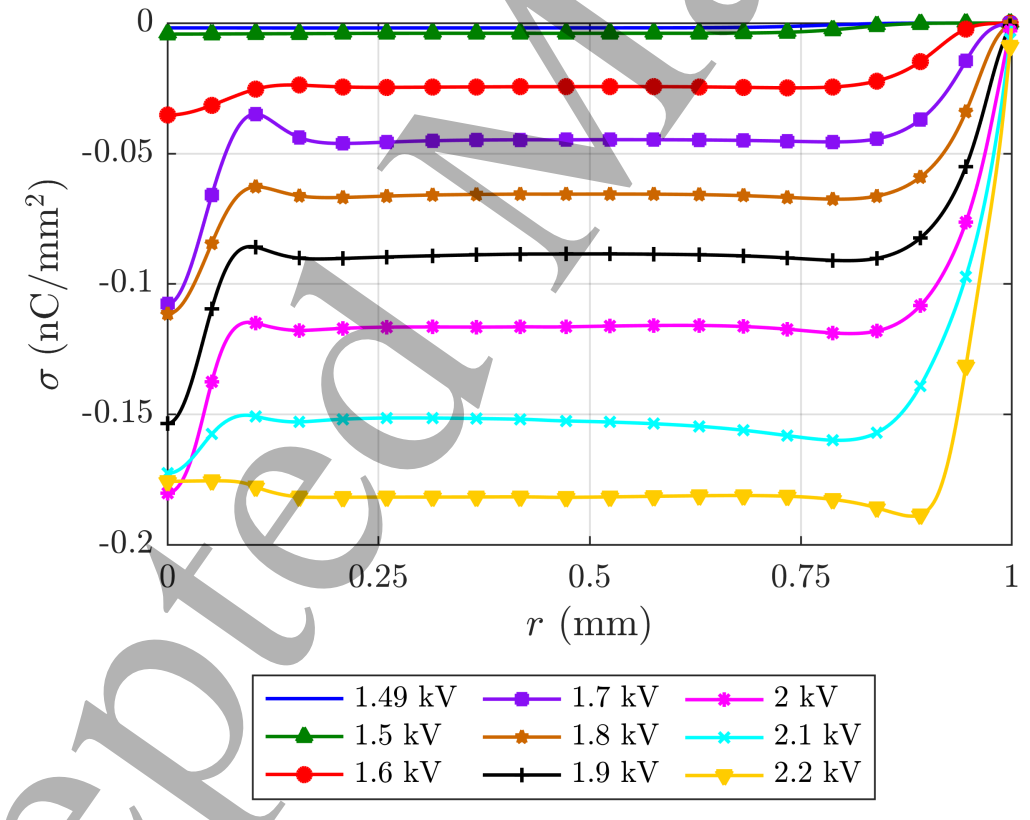

(b)

Figure 11: Surface charge density on the void surface after the discharge had finished at different applied voltages magnitudes.

The PD activity model developed using these plasma simulations treats the void as a single object undergoing discharges. When a discharge occurs it reduces the electric field in the void to a residual value, which is dependent on the magnitude of the electric field before the discharge, and results in an apparent charge across the measuring ground electrode. The residual field is typically assumed to be a constant [4, 5]. However, 
the plasma simulations show that the mean field in the void after a PD is dependent on the magnitude of the electric field before the PD, see Figure 12 . This demonstrates the power of using simulations of plasma dynamics to inform PD activity models.

It is known that surface charge on the surface of the void before a PD occurs will influence plasma dynamics and surface charge deployed by that PD [9]. The space charge from previous PDs within the air is assumed to be removed between PD events due to recombination and the deployment of charged particles at the void boundary due to the changing applied field. Canonical reasoning, and inyestigations using a simple one dimensional plasma dynamics model [28], suggest that charged particles are completely removed from the gas between discharges. The influence of surface charge on PD activity is neglected in the model introduced in this work for two reasons. Firstly, to properly determine the influence of charge already on the void surface would require a significantly larger number of plasma dynamic simulations, and would add to the complexity of the PD activity model. More importantly, the rate of seed charge generation is uncertain, as will be seen in the following section, which is likely to more significantly impact the results. The view was taken that implementing a complex method of simulating the influence of surface charge density on PD is unlikely to add value to the model. The data required for the PD activity model is displayed in Figures 12 and 13 .

Figure 12: Relationship between the electric field before PD and the electric field after PD. 
Figure 13: Relationship between the electric field before PD and the apparent charge of the PD.

\section{$5 \quad$ Partial Discharge Activity Model}

The simulations of plasma dynamics are used to implement a simple PD activity model. PD activity is assumed to be dependent on the mean electric field within the void, $E_{\text {mean }}$, which is a sum of the mean applied electric field and the mean local electric field from surface charge at the void boundary. The influence of charge already on the void surface is ignored, with the exception of its influence on the mean electric field, such that discharges can be treated as a superposition of discharges within virgin voids. Following the literature a PD occurs at a given time if the electrie field is sufficiently high

and a free electron is available

$$
\left|E_{\text {mean }}\right|>E_{\text {inc }}
$$

$$
\dot{N}_{\mathrm{e}} \Delta t>R
$$

where $\dot{N}_{\mathrm{e}}$ is the electron generation rate, $\Delta t$ is the simulation time step and $R$ is a random number uniformly distributed between 0 and 1 . A PD reduces the $E_{\text {mean }}$ to a residual value, and produces an apparent charge on the measuring ground electrode. Based on the investigations performed in the previous section $E_{\text {inc }}=7.97 \mathrm{kV} / \mathrm{mm}$. The remaining data required for the PD activity model is displayed in Figures 12 and 13. The model of PD activity is similar to existing work in the literature, [5], the difference is that is informed by a plasma dynamics model meaning that the residual electric field and the surface charge distributions do not need to be treated as free parameters.

In order to simulate PD activity it is necessary to develop an expression for the electron generation rate. Between $\mathrm{PD}$ events the charged particles are removed from the air due to recombination and charge deployment at the void boundaries due to the changing applied field. Canonical reasoning, and investigations using a simple one dimensional plasma dynamics model [28], suggest that charged particles are completely removed from the gas between discharges. The two main mechanisms that can generate free charge in the void are background radiation within the gas ionising a neutral molecule and charge emission from trapped charge on the dielectric surface from earlier PDs. Of these two processes, surface emission is by far the most dominant and background radiation will be neglected in the model of PD activity.

The surface emission of trapped charge from dielectric interfaces is poorly understood. In [11] the electron emission from a dielectric surface was assumed to obey the Richardson-Schottky law. However, it is not clear whether this is valid for a layer of trapped charge on a dielectric surface. The seminal work of Niemeyer 
used an equation, which was subsequently adopted in much of the PD activity modelling literature, where the electron generation rate for trapped charge on a dielectric surface is dependent on the amout of charge deployed by the most recent PD combined with a work function and Schottky term 4 . This is expression is based on earlier work measuring electron emission from concentric glass tubes in a vacuum under DC voltage 29]. However, in this earlier work the potential barrier in the Schottky term is doubled because when charge is emitted from an insulator an immobile hole remains, as opposed to when charge is emitted from a metallic surface where the interaction is between an electron and an image charge [29]. It is clear that all of these approaches are an imperfect way of simulating seed charge generation for PD. In this work a simple approach is used, with $\dot{N}_{\mathrm{e}}$ set to

$$
\dot{N}_{\mathrm{e}}=c_{\mathrm{e}} \exp \left(\frac{\sqrt{\frac{e^{3}\left|E_{\text {mean }}\right|}{2 \pi \varepsilon_{0}}}}{k_{\mathrm{B}} T}\right),
$$

where $c_{\mathrm{e}}$ is an adjustable free parameter with units of $\mathrm{s}^{-1}, k_{\mathrm{B}}$ is the Boltzmann constant and $T=300 \mathrm{~K}$ is the temperature. (19) uses the Schottky term adjusted for insulator surfaces in accordance with the earlier work [29]. $c_{\mathrm{e}}$ is treated a single value, which is adjusted such that the simulation matches the experimental data. It should be realised that in practice $c_{\mathrm{e}}$ depends on a large number of variables including, but not limited to: the phonon frequency of LDPE, the work function of LDPE and the detrappable charge at the dielectric boundary. The precise value of these variables is unknown, as is their impact on $\dot{N}_{\mathrm{e}}$. The simple approach taken here is to use a lumped adjustable parameter $c_{\mathrm{e}}$.

The PD activity model was implemented in MATLAB, due to its simplicity thousands of PDs can be simulated within minutes. A comparison of PDs per cycle and mean PD magnitude between simulation and experiment is provided in Figure 14. The results show some discrepancy between measurement and simulation, there are a numerous reasons that could explain this. Measured PD activity could be impacted by minute variations in the sample fabrication, despite efforts to maintain homogeneity, and changing environmental conditions including humidity and ambient temperature. It is also possible that many low magnitude PDs could be 'lost' in the noise floor, resulting in a lower number of PDs per cycle. From a simulation viewpoint, a simple model is used which assumes $\mathrm{PD}$ is solely dependent on the mean electric field. Another point is that the drift diffusion model uses swarm parameters to capture the dynamics of the charged species. Alternative values for swarm parameters in air have been used by other researchers, and could result in slightly different PD magnitudes. A more fundamental issue is that the generation of seed charge for PD in voids bounded by a dielectric material is poorly understood. It should be noted that adjusting the expression for $\dot{N}_{\mathrm{e}}$, and introducing additional free parameters, would result in a better fit with experimental data, but it would require making additional assumptions with limited justification. Many of the PD activity models introduced in the literature would be able to provide a better fit to the experimental data than that shown here, but would require a larger number of free parameters.

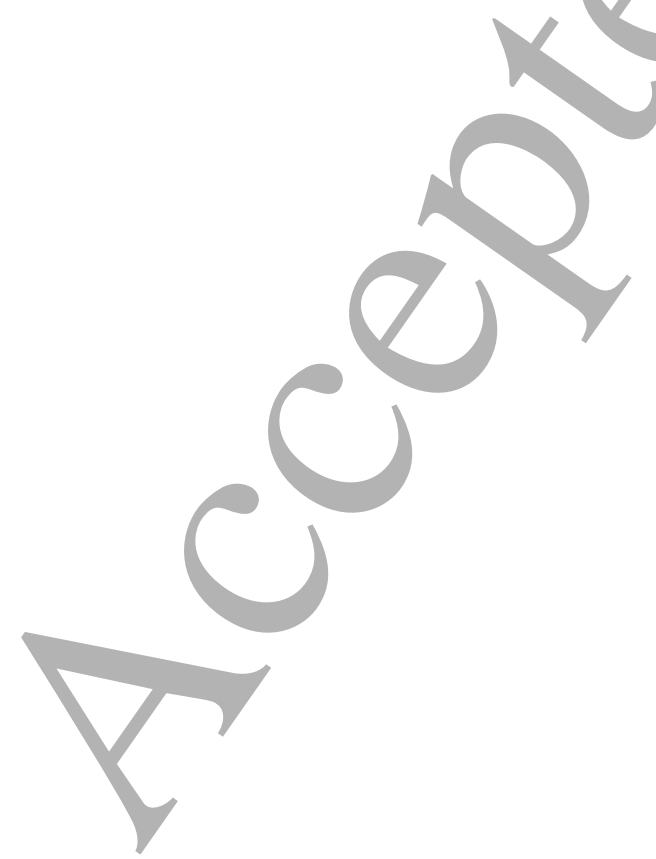




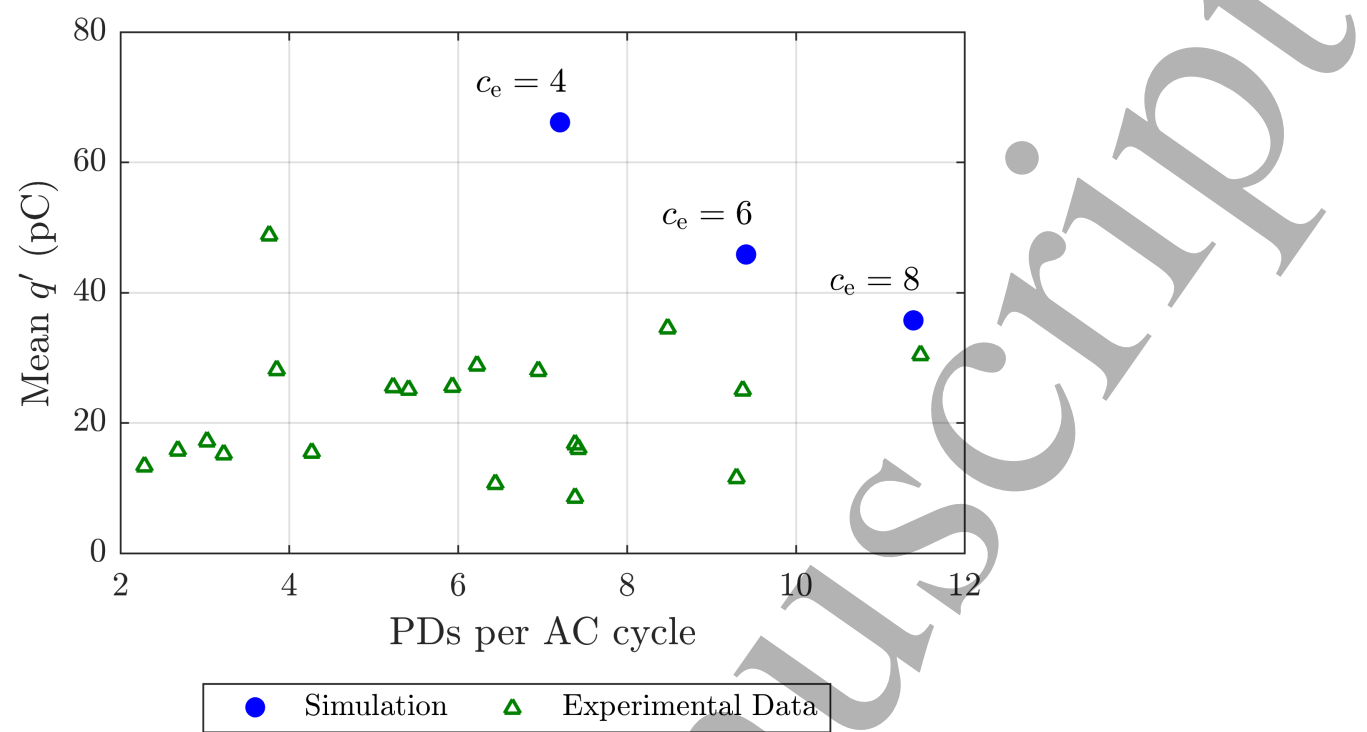

Figure 14: A scatter plot of PD statistical quantities from experimental samples and the PD activity model for different values of $c_{\mathrm{e}}$.

A typical PRPD pattern from the simulation is shown in Figure 15. It is visually similar to many of the experimental PRPD patterns, see Figure 3 although it has a higher number of PDs per cycle compared to experiment.

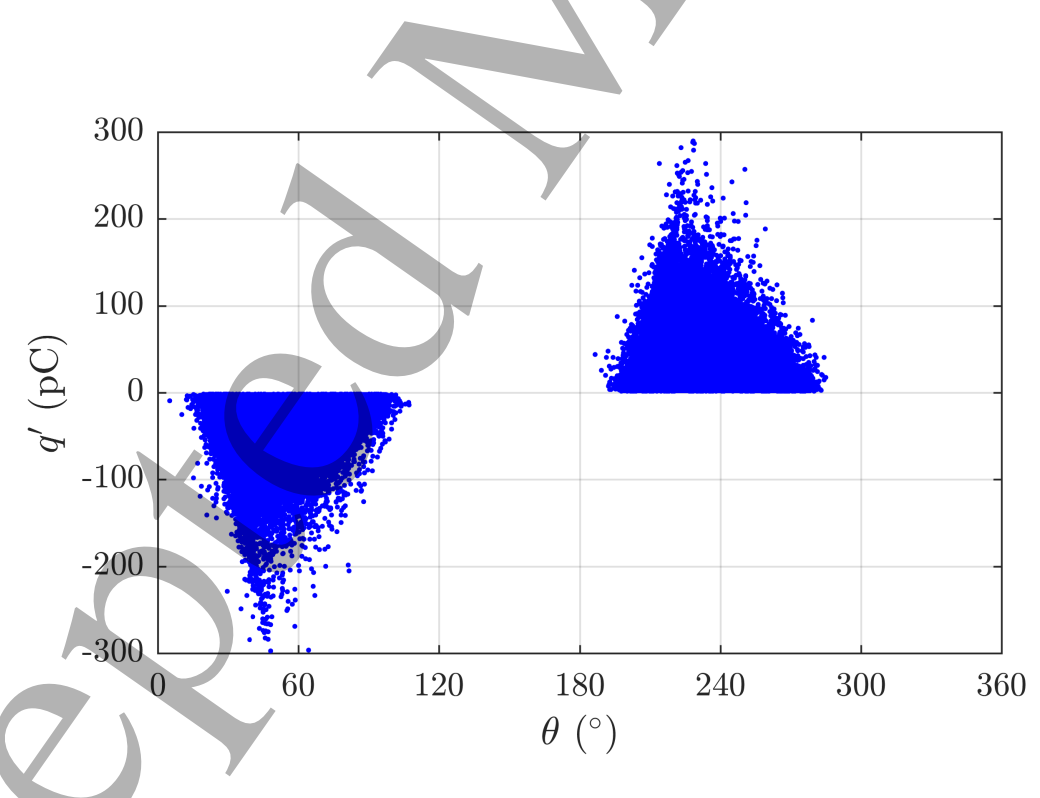

Figure 15: Simulated PRPD pattern for $c_{\mathrm{e}}=8$. The $50 \mathrm{~Hz} \mathrm{AC}$ applied voltage RMS is set to the mean experimental PDIV value of $1.49 \mathrm{kV}$.

It can be concluded with confidence that PD activity consists of low magnitude discharges with occasional large magnitude discharges, both of which deposit charge over the majority of the void surface. It is expected that during this initial stage, damage could occur over the entire surface of the void. This agrees with 

which would lead to discharges occurring within a nitrogen environment. This would inhibit photoionisation processes, and is likely to lead to discharges which do not spread over the entire void surface. This would explain the formation of localised pits on the dielectric surface [30. From an electrical engineering perspective, plasma dynamic simulations allow estimates on the magnitude of PD, insight into the potential damage it could do to the surface of the void and the apparent current pulses a discharge will generate.

\section{Conclusions}

A PD activity model was developed for the initial stages of discharge activity within a cylindrical void surrounded by LDPE. For the experimental arrangement considered discharge activity could be simulated using a single free parameter, $c_{\mathrm{e}}$, which was used to adjust the rate of seed charge generation. This work demonstrates that simulations of plasma dynamics can be used to inform PD activity models and allows the development of models with a reduced number of free parameters. The specific findings off this work are as follows:

- The inception electric field, the minimum electric field required for the void to discharge, was in good agreement with experimental data, with a predicted inception voltage of $1.49 \mathrm{kV}$ compared to a mean experimental value of $1.57 \mathrm{kV}$.

- The electric field in the void after PD, commonly referred to as the residual field, is not a single value as is commonly assumed [4, 5. In the geometry considered discharges that just exceeded the inception electric field resulted in high residual fields, whereas discharges are higher electric fields resulted in a significantly reduced electric field, see Figure 12 .

- The PD activity model was in reasonable agreement with the measured PD data, which is an encouraging result as only a single adjustable parameter was used.

- An issue which should be addressed moving forwards is the generation of seed charge for PDs. PDs often occur at electric fields that significantly exceed the inception conditions of the void. This work, following the literature, treated seed charge generation as stochastic process, with a probability dependent on the electron generation rate. This is a simplified technique of describing what is a complex process, and improvements in measurement and underlying theory are required.

\section{Acknowledgments}

The authors acknowledge the use of the IRIDIS High Performance Computing Facility and associated support services at the University of Southampton. This work was supported by an EPSRC Doctoral Training Centre grant (EP/G03690X/1) and by a Royal Thai Government Scholarship.

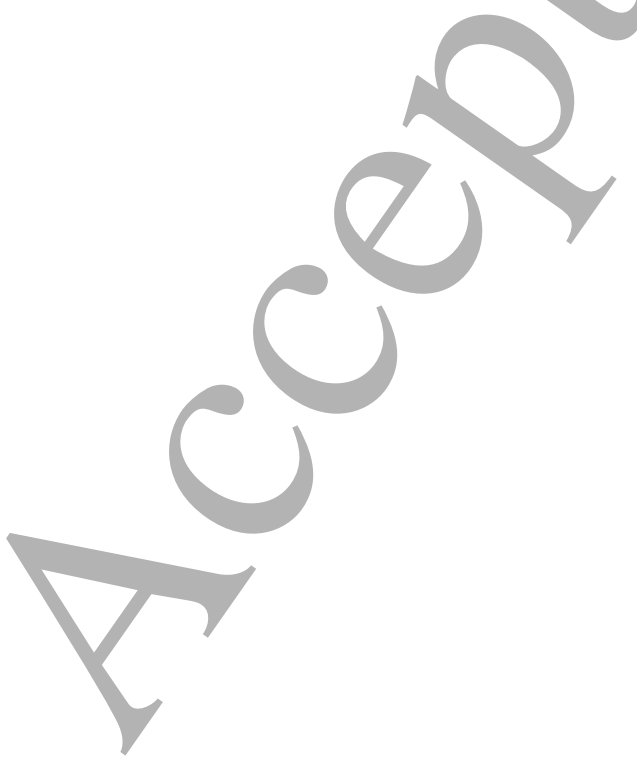




\section{Appendix A}

The swarm parameters for air are those introduced by Morrow and Lowke [22. They are explicitly

$$
\begin{aligned}
& \alpha, \mathrm{cm}^{-1}= \begin{cases}2 \times 10^{-16} N \exp \left(-7.248 \times 10^{-15} /(E / N)\right) & \text { if } E / N>1.5 \times 10^{-15} \\
6.619 \times 10^{-17} N \exp \left(-5.593 \times 10^{-15} /(E / N)\right) & \text { if } E / N \leq 1.5 \times 10^{-15}\end{cases} \\
& \eta, \mathrm{cm}^{-1}=\left\{\begin{array}{cc}
N\left[8.889 \times 10^{-5}(E / N)+2.567 \times 10^{-19}\right]+\ldots & \text { if } E / N>1.05 \times 10^{-15} \\
N^{2}\left[4.7778 \times 10^{-59}(E / N)^{-1.2749}\right] & \\
N\left[6.089 \times 10^{-4}(E / N)-2.893 \times 10^{-19}\right]+\ldots & \text { if } E / N \geq 1.2 \times 10^{-17} \\
N^{2}\left[4.7778 \times 10^{-59}(E / N)^{-1.2749}\right] & E / N \leq 1.05 \times 10^{-15} \\
106.81 & \text { if } E / N<1.2 \times 10^{-17} \\
0 & \text { if } \eta<0
\end{array}\right.
\end{aligned}
$$

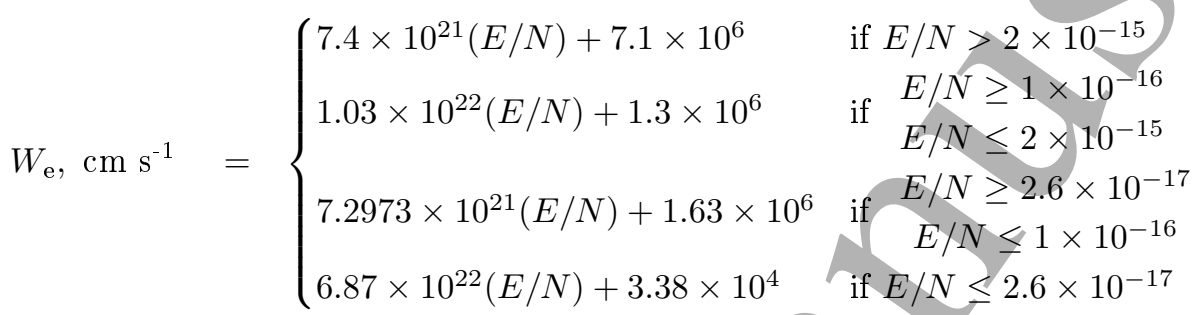

$$
\begin{aligned}
& W_{\mathrm{p}}, \mathrm{cm} \mathrm{s}^{-1}=2.43 E P_{0} / P \\
& W_{\mathrm{n}}, \mathrm{cm} \mathrm{s}^{-1}= \begin{cases}2.7 E P_{0} / P & \text { if } E / N>5 \times 10^{-16} \\
1.86 E P_{0} / P & \text { if } E / N \leq 5 \times 10^{-16}\end{cases} \\
& \beta_{\mathrm{ep}}, \mathrm{cm}^{3} \mathrm{~s}^{-1}=2 \times 10^{-7} \\
& \beta_{\mathrm{np}}, \mathrm{cm}^{3} \mathrm{~s}^{-1}=2 \times 10^{-7} \\
& D_{\mathrm{e}}, \mathrm{cm}^{2} \mathrm{~s}^{-1}=\left[0.3341 \times 10^{9}(E / N)^{0.54069}\right]\left(W_{\mathrm{e}} / E\right)
\end{aligned}
$$

where $E$ is the electric field magnitude in units of $\mathrm{V} / \mathrm{cm}$ and $N$ is the number density of gas molecules per unity volume in units of $\mathrm{cm}^{-3}, P$ is the air pressure in Torr and $P_{0}$ is atmospheric pressure in Torr. The magnitude of the charged species drift velocities are provided in (22)-(24). The drift velocity vector is aligned in the direction of the electric field for positive ions and opposite the electric field for negative ions and electrons.

\section{References}

[1] R.J. Van Brunt. Physics and chemistry of partial discharge and corona. recent advances and future challenges. IEEE Transactions on Dielectrics and Electrical Insulation, 1(5):761-784, 1994.

[2] G.C. Montanari. Aging and life models for insulation systems based on pd detection. IEEE Transactions on Dielectrics and Electrical Insulation, 2(4):667-675, 1995.

[3] L. Niemeyer, B. Fruth, and F. Gutfleisch. Simulation of partial discharges in insulation systems. In High Voltage Engineering, 1991. 7th International Symposium on, pages 25-28, 1991.

[4] L. Niemeyer. A generalized approach to partial discharge modeling. IEEE Transactions on Dielectrics and Electrical Insulation, 2(4):510-528, 1995.

[5] H.A. Illias, G. Chen, and P.L. Lewin. The influence of spherical cavity surface charge distribution on the sequence of partial discharge events. Journal of Physics D: Applied Physics, 44(24):245202, 2011.

[6] C. Forssen and H. Edin. Partial discharges in a cavity at variable applied frequency part 2: measurements and modeling. IEEE Transactions on Dielectrics and Electrical Insulation, 15(6):1610-1616, 2008.

[7] G.C. Crichton, P.W. Karlsson, and A. Pedersen. Partial discharges in ellipsoidal and spheroidal voids. IEEE Transactions on Electrical Insulation, 24(2):335-342, 1989. 
[8] H.A. Illias, G. Chen, and P.L. Lewin. Partial discharge behavior within a spherical cavity in a solid dielectric material as a function of frequency and amplitude of the applied voltage. IEEE Transactions on Dielectrics and Electrical Insulation, 18(2):432-443, 2011.

[9] G. Callender, I.O. Golosnoy, P. Rapisarda, and P.L. Lewin. Critical analysis of partial discharge dynamics in air filled spherical voids. Journal of Physics D: Applied Physics, 51(12):125601, 2018.

[10] G.E. Georghiou, A.P. Papadakis, R. Morrow, and A.C. Metaxas. Numerical modelling of atmospheric pressure gas discharges leading to plasma production. Journal of Physics D: Applied Physics, 38(20):303, 2005.

[11] A. Villa, L. Barbieri, M. Gondola, A.R. Leon-Garzon, and R. Malgesini. A pde-based partial discharge simulator. Journal of Computational Physics, 345:687-705, 2017.

[12] C. Pan, K. Wu, Y. Du, J. Tang, X. Tao, and Y. Luo. Simulation of cavity pd sequences at dc voltage by considering surface charge decay. Journal of Physics D: Applied Physics, 50(20):205202, 2017.

[13] C. Pan, Y. Meng, K. Wu, Z. Han, K. Qin, and Y. Cheng. Simulation of partial discharge sequences using fluid equations. Journal of Physics D: Applied Physics, 44(25):255201, 2011.

[14] N.Y. Babaeva and M.J. Kushner. Self-organization of single filaments and diffusive plasmas during a single pulse in dielectric-barrier discharges. Plasma Sources Science and Technology, 23(6):065047, 2014.

[15] Th. Callegari, R. Ganter, and J.P. Boeuf. Diagnostics and modeling of a macroscopic plasma display panel cell. Journal of Applied Physics, 88(7):3905-3913, 2000.

[16] T. Tanmaneeprasert and P. L. Lewin. Electrical treeing and ageing characteristics in cavities of low density polyethylene dielectrics on partial discharge measurements. In 2016 IEEE Conference on Electrical Insulation and Dielectric Phenomena (CEIDP), pages 975-978, Oct 2016.

[17] P.H.F Morshuis. Partial discharge mechanisms. PhD thesis, Delft University of Technology, 1993.

[18] Partial discharge measurements. IEC 60270, 1998.

[19] H.A. Illias, G. Chen, A.H.A. Bakar, H. Mokhlis, and M.A. Tunio. Partial discharges within two spherical voids in an epoxy resin. Journal of Physics D: Applied Physics, 46(33):335301, 2013.

[20] P. Dordizadeh, K. Adamiak, and G.S. Peter Castle. Numerical investigation of the formation of trichel pulses in a needle-plane geometry. Journal of Physics D: Applied Physics, 48(41):415203, 2015.

[21] P. Dordizadeh, K. Adamiak, and G.S. Peter Castle. Study of the impact of photoionization on negative and positive needle-plane corona discharge in atmospheric air. Plasma Sources Science and Technology, 25(6):065009, 2016.

[22] R. Morrow and J.J. Lowke. Streamer propagation in air. Journal of Physics D: Applied Physics, 30(4):614, 1997.

[23] A. Bourdon, V.P. Pasko, N.Y. Liu, S. Célestin, P. Ségur, and E. Marode. Efficient models for photoionization produced by non-thermal gas discharges in air based on radiative transfer and the helmholtz equations. Plasma Sources Science and Technology, 16(3):656, 2007.

[24] M.B. Zhelezniak, A.K. Mnatsakanian, and S.V. Sizykh. Photoionization of nitrogen and oxygen mixtures by radiation from a gas discharge. High Temperature Science, 20(6):357-362, 1982.

[25] COMSOL Multiphysics Version 5.3 reference manual.

[26] K. Wu, C. Pan, Y. Meng, and Y. Cheng. Dynamic behavior of surface charge distribution during partial discharge sequences. IEEE Transactions on Dielectrics and Electrical Insulation, 20(2):612-619, 2013.

[27] F. Gutfleisch and L. Niemeyer. Measurement and simulation of PD in epoxy voids. IEEE Transactions on Dielectrics and Electrical Insulation, 2(5):729-743, 1995.

[28] G. Callender. Modelling Partial Discharge in Gaseous Voids. PhD thesis, University of Southampton, 2018.

[29] D.B. Hibbert and A.J.B. Robertson. The emission of electrons from glass induced by a strong electric field and the mechanism of the silent electric discharge. Proceedings of the Royal Society of London A: Mathematical, Physical and Engineering Sciences, 349(1656):63-79, 1976.

[30] T. Tanaka. Internal partial discharge and material degradation. IEEE Transactions on Electrical Insulation, 21 (6):899-905, Dec 1986. ISSN 0018-9367. 